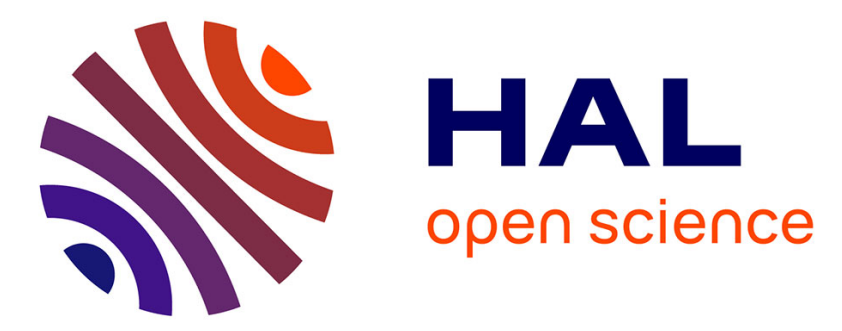

\title{
Discovery of a Spin-down State Change in the LMC Pulsar B0540-69
}

F. E. Marshall, Lucas Guillemot, A. K. Harding, P. Martin, D. A. Smith

\section{To cite this version:}

F. E. Marshall, Lucas Guillemot, A. K. Harding, P. Martin, D. A. Smith. Discovery of a Spin-down State Change in the LMC Pulsar B0540-69. The Astrophysical journal letters, 2015, 807 (2), pp.L27. 10.1088/2041-8205/807/2/L27 . insu-01182158

\section{HAL Id: insu-01182158 https://hal-insu.archives-ouvertes.fr/insu-01182158}

Submitted on 9 Jun 2016

HAL is a multi-disciplinary open access archive for the deposit and dissemination of scientific research documents, whether they are published or not. The documents may come from teaching and research institutions in France or abroad, or from public or private research centers.
L'archive ouverte pluridisciplinaire HAL, est destinée au dépôt et à la diffusion de documents scientifiques de niveau recherche, publiés ou non, émanant des établissements d'enseignement et de recherche français ou étrangers, des laboratoires publics ou privés. 


\title{
DISCOVERY OF A SPIN-DOWN STATE CHANGE IN THE LMC PULSAR B0540-69
}

\author{
F. E. Marshall ${ }^{1}$, L. Guillemot ${ }^{2,3}$, A. K. Harding $^{1}$, P. Martin ${ }^{4}$, and D. A. Smith ${ }^{5}$ \\ ${ }^{1}$ Astrophysics Science Division, NASA Goddard Space Flight Center, Greenbelt, MD 20771, USA; frank.marshall@ nasa.gov \\ ${ }^{2}$ Laboratoire de Physique et Chimie de l'Environnement et de l'Espace (LPC2E), CNRS-Université d'Orléans, F-45071 Orléans, France \\ ${ }^{3}$ Station de radioastronomie de Nançay, Observatoire de Paris, CNRS/INSU F-18330 Nançay, France \\ ${ }^{4}$ Institut de Recherche en Astrophysique et Planétologie, UPS/CNRS, UMR5277, F-31028 Toulouse Cedex 4, France \\ ${ }^{5}$ Centre d'Études Nucléaires de Bordeaux Gradignan, IN2P3/CNRS, Université de Bordeaux 1, BP120, F-33175, Gradignan Cedex, France \\ Received 2015 May 19; accepted 2015 June 17; published 2015 July 8
}

\begin{abstract}
We report the discovery of a large, sudden, and persistent increase in the spin-down rate of B0540-69, a young pulsar in the Large Magellanic Cloud, using observations from the Swift and RXTE satellites. The relative increase in the spin-down rate $\dot{\nu}$ of $36 \%$ is unprecedented for B0540-69. No accompanying change in the spin rate is seen, and no change is seen in the pulsed X-ray emission from B0540-69 following the change in the spin-down rate. Such large relative changes in the spin-down rate are seen in the recently discovered class of "intermittent pulsars," and we compare the properties of B0540-69 to such pulsars. We consider possible changes in the magnetosphere of the pulsar that could cause such a large change in the spin-down rate.
\end{abstract}

Key words: pulsars: individual (PSR B0540-69)

\section{INTRODUCTION}

B0540-69 is a young, rotationally powered pulsar in the Large Magellanic Cloud (LMC) that is similar to the Crab pulsar in many ways. With a rotation frequency $\nu$ of almost $20 \mathrm{~Hz}$, it is one of the most rapidly rotating young pulsars known. Its spin-down luminosity $\left(-4 \pi^{2} I \nu \dot{\nu}\right)$ of $\sim 1.5 \times 10^{38} I_{45}$ $\mathrm{erg} \mathrm{s}^{-1}$, where $I_{45}$ is the moment of inertia of the neutron star (NS) in units of $10^{45} \mathrm{~g} \mathrm{~cm}^{2}$, is also among the largest for all pulsars. The spin-down rate of a pulsar is often described in terms of a braking index $n$, in which $\dot{\nu}=-\kappa \nu^{n}$. The slowdown of B0540-69 is relatively stable for a young pulsar, and it is one of only eight young pulsars for which a braking index has been reliably measured (Lyne et al. 2015). It has a characteristic spin-down age $(-\nu / 2 \dot{\nu})$ of $\sim 1600$ years.

Pulsars are remarkably stable rotators, and the deviations from clocklike precision can provide information about the structure and processes at work in the NS and its magnetosphere. Sudden changes in $\nu$ and $\dot{\nu}$ ("glitches") are occasionally seen in pulsars, especially those with ages between $10^{3}$ and $10^{5}$ years ( $\mathrm{Yu}$ et al. 2013). Glitches are thought to occur in rotationally powered pulsars when angular momentum is transferred from a more rapidly rotating component of the NS to the outer crust (e.g., Anderson \& Itoh 1975; Franco et al. 2000). A different kind of rapid change is seen in some pulsars that affects the spin-down rate. In their examination of longterm monitoring of 366 pulsars, Lyne et al. (2010) found that many pulsars rapidly switch between two different spin-down rates. The timescales for the transitions are quasi-periodic, with typical timescales of years. "Intermittent pulsars" are extreme examples of such pulsars. They transition from a radio-on state to radio-off state with a simultaneous change in the spin-down rate. The best studied example is PSR B1931+24 (Kramer et al. 2006; Young et al. 2013) for which multiple transitions on a timescale of weeks from a radio bright state with $\dot{\nu}=-16 \times 10^{-15} \mathrm{~Hz} \mathrm{~s}^{-1}$ and a radio quiet state with $\dot{\nu}=-10.8 \times 10^{-15} \mathrm{~Hz} \mathrm{~s}^{-1}$ have been observed. Similar behavior on longer timescales has been reported for PSR J1832 +0029 (Lorimer et al. 2012) and PSR J1841-0500 (Camilo et al. 2012). All three pulsars show rapid transitions between two states with stable spin-down rates, large differences in the spin-down rates, large changes in the radio flux, and no simultaneous change in $\nu$. Unlike most explanations for glitches, models for these state changes have emphasized changes in the pulsar's magnetosphere (e.g., Li et al. 2012). None of the intermittent pulsars have been detected in the optical, X-ray, or gamma-ray bands, but somewhat similar behavior has been seen for the gamma-ray pulsar PSR J2021 +4026 (Allafort et al. 2013). A 4\% increase in $\dot{\nu}$ was seen with a simultaneous decrease of $18 \%$ in the flux above $100 \mathrm{MeV}$ with a timescale for the transition of less than a week. Since there is no radio counterpart, any change in radio flux is not known.

B0540-69 has been extensively studied since its discovery with the Einstein Observatory (Seward et al. 1984). A small glitch for B0540-69 was reported by Zhang et al. (2001) with a relative change in $\nu$ of $1.9 \times 10^{-9}$ and $\dot{\nu}$ of $8.5 \times 10^{-5}$. The reality of this glitch was disputed by Cusumano et al. (2003) and later supported by Livingstone et al. (2005). Ferdman et al. (2015) examined 15.8 years of data from RXTE and reported a second glitch with a relative change in $\nu$ of $1.6 \times 10^{-9}$ and $\dot{\nu}$ of $9.3 \times 10^{-5}$. Both these changes in $\dot{\nu}$ are orders of magnitude smaller than we report in this paper. No other glitches have been reported in the extensive monitoring of the pulsar. Optical pulsations are also seen (Mignani et al. 2010 and references therein). Manchester et al. (1993) discovered radio pulsations with an above average luminosity at $640 \mathrm{MHz}$ of $1200 \mathrm{mJy} \mathrm{kpc}{ }^{2}$.

In this paper we present and discuss new timing analysis of B0540-69 as observed with RXTE and Swift. Details of the observations and the results of the temporal analysis are reported in Section 2, and interpretations are given in Section 3. Finally, Section 4 is a summary.

\section{OBSERVATIONS AND DATA REDUCTION}

B0540-69 was observed with the Proportional Counter Array (PCA) on board the RXTE (Bradt et al. 1993) during a 12.5 year campaign to monitor the nearby PSR J0537-6910. The final observation was on 2011 December 31. We 
Table 1

Observing Log

\begin{tabular}{|c|c|c|c|c|}
\hline Satellite & Obs. ID & Date $^{\mathrm{a}}$ & $\mathrm{MJD}^{\mathrm{a}}$ & $T_{\exp }{ }^{\mathrm{b}}$ \\
\hline$R X T E$ & $96023-01-17$ & 2011 Aug 13 & 55786 & 6.6 \\
\hline$R X T E$ & $96023-01-42$ & 2011 Sep 06 & 55810 & 7.3 \\
\hline$R X T E$ & $96023-01-43$ & 2011 Sep 14 & 55818 & 7.5 \\
\hline$R X T E$ & $96023-01-44$ & 2011 Sep 18 & 55822 & 6.9 \\
\hline$R X T E$ & $96023-01-20$ & 2011 Sep 23 & 55827 & 6.9 \\
\hline$R X T E$ & $96023-01-45$ & 2011 Sep 26 & 55830 & 7.2 \\
\hline$R X T E$ & $96023-01-46$ & 2011 Sep 26 & 55830 & 6.3 \\
\hline$R X T E$ & $96023-01-21$ & 2011 Oct 09 & 55843 & 6.7 \\
\hline$R X T E$ & $96023-01-22$ & 2011 Oct 22 & 55856 & 6.9 \\
\hline$R X T E$ & $96023-01-23$ & 2011 Nov 04 & 55869 & 6.9 \\
\hline$R X T E$ & $96023-01-24$ & 2011 Nov 18 & 55883 & 7.3 \\
\hline$R X T E$ & $96023-01-25$ & 2011 Dec 03 & 55898 & 6.6 \\
\hline$R X T E$ & $96023-01-26$ & 2011 Dec 17 & 55912 & 7.1 \\
\hline$R X T E$ & 96023-01-19 & 2011 Dec 31 & 55926 & 7.8 \\
\hline Swift & 00033603002 & 2015 Feb 17 & 57070 & 1.2 \\
\hline Swift & 00033603004 & 2015 Feb 23 & 57077 & 2.1 \\
\hline Swift & 00033603005 & 2015 Feb 25 & 57078 & 1.8 \\
\hline Swift & 00033603006 & 2015 Feb 25 & 57078 & 2.4 \\
\hline Swift & 00033603007 & 2015 Mar 11 & 57092 & 2.1 \\
\hline Swift & 00033603008 & 2015 Apr 11 & 57123 & 2.3 \\
\hline Swift & 00033603009 & 2015 Apr 13 & 57125 & 2.3 \\
\hline Swift & 00033603010 & 2015 Apr 23 & 57135 & 2.5 \\
\hline
\end{tabular}

Notes.

a At the start of the observation.

b Exposure time in ks.

concentrate on observations covering the final 140 days of the campaign. Results from RXTE observations have been reported by Zhang et al. (2001), Livingstone et al. (2005) and Ferdman et al. (2015). The PCA is composed of five co-aligned xenon detectors (proportional counter units (PCUs)) with a total area of $\sim 6500 \mathrm{~cm}^{2}$. Individual PCUs were routinely turned off and on to reduce the number of high-voltage breakdowns. Additional observations were made with the X-ray telescope (XRT) instrument (Burrows et al. 2005) on the Swift GammaRay Burst Explorer (Gehrels et al. 2004) starting in 2015 February. Table 1 is a log of the observations.

Data reduction for both missions followed very similar procedures. X-ray events were screened to maximize the signal-to-noise ratio $(\mathrm{S} / \mathrm{N})$, and then photon arrival times were corrected to the solar system barycenter with the FTOOL $^{6}$ FAXBARY for RXTE and BARYCORR for Swift using the Hubble Space Telescope position of $\alpha=05^{\mathrm{h}} 40^{\mathrm{m}} 11.202$, $\delta=-69^{\circ} 19^{\prime} 54^{\prime \prime} 17$ (J2000.0) (Mignani et al. 2010). The mid-point of each observation was chosen as the epoch, and then the best period was determined by comparing multiple folded light curves using EFSEARCH. Since the folded light curve is approximately a sine wave (Cusumano et al. 2003), the phase at the epoch was determined by fitting a sine wave to the folded light curve produced with EFOLD and using the phase of the peak of the sine wave. The resulting frequencies and phases were fit to the usual truncated Taylor series expansion of the phase and its time derivatives $\nu, \dot{\nu}$, and $\ddot{\nu}$.

Uncertainties quoted in this paper are given at the $90 \%$ confidence level unless otherwise noted. For multi-parameter fits, the other parameters are allowed to vary when calculating the limits.

\footnotetext{
6 http://heasarc.gsfc.nasa.gov/docs/software/lheasoft/
}

\subsection{RXTE/PCA Observations}

The PCA is sensitive to X-rays in the $2-60 \mathrm{keV}$ band with moderate $(\Delta E / E \sim 18 \%)$ resolution. Each event is timetagged on the spacecraft with an accuracy better than $5 \times 10^{-5} \mathrm{~s}$ (Rots et al. 1998). To improve the $\mathrm{S} / \mathrm{N}$, only events in the first xenon layer in the energy range of 3-20 keV were included.

Figure 1 shows the frequency residuals for the last 14 $R X T E$ observations relative to the best-fit ephemeris model for the first 12 of the observations. The value of $\ddot{\nu}$ is set to $3.249 \times 10^{-21} \mathrm{~s}^{-3}$, the best-fit value for observations between 2010 October and 2011 December. The model, whose parameters are given in Table 2, provides a very good fit to the first 12 observations with rms phase residuals of $\sim 1 \%$, but the final two observations deviate dramatically. A linear fit to the frequencies for the final two observations indicates that $\dot{\nu}$ has changed by $-6.0 \times 10^{-11} \mathrm{~Hz} \mathrm{~s}^{-1}$ with a statistical uncertainty of $0.8 \times 10^{-11} \mathrm{~Hz} \mathrm{~s}^{-1}$ at some time between the observations on December 3 and 17. This is an increase of $32 \%$ $\pm 4 \%$ in the rate at which the pulsar's spin is slowing. This value is consistent with, but less accurate than, the value determined in Section 2.2 using both Swift and RXTE data. We note that our value for $\ddot{\nu}$ is $\sim 14 \%$ lower than the values reported by Ferdman et al. (2015) for longer intervals of RXTE data. Reprocessing the RXTE data using the Ferdman et al. values for $\ddot{\nu}$ demonstrates that revising $\ddot{\nu}$ would have a negligible effect on our results and would not change our conclusions.

If the sudden change in $\dot{\nu}$ were due to a glitch, then a simultaneous change in $\nu$ would be expected. Surveys of glitches in other pulsars find that the amplitude of a glitch in $\nu$ correlates with the amplitude of the glitch in $\dot{\nu}$ for pulsars in general (Espinoza et al. 2011) and for the Crab Pulsar in particular (Lyne et al. 2015). The correlation for pulsars in general would indicate an accompanying glitch in B054069 larger than $\sim 1 \times 10^{-5} \mathrm{~Hz}$, and the correlation for the Crab would indicate an accompanying glitch of $\sim 1 \times 10^{-3} \mathrm{~Hz}$. There is no indication of an accompanying change in $\nu$, and we place limits on its possible size by assuming an instantaneous glitch with no recovery and extrapolating the fit to the postglitch frequencies back toward the final pre-glitch observation. With these assumptions the largest glitch would occur immediately after the final pre-glitch observation with a size of $1.2 \times 10^{-5} \mathrm{~Hz}$. Later glitches would be smaller until at $200 \mathrm{ks}$ after final pre-glitch observation there would be no glitch in $\nu$.

Large glitches in $\dot{\nu}$ typically decay away on timescales from about a day to weeks. Since the new value for $\dot{\nu}$ is consistent with that later seen with Swift (Section 2.2), there is also no indication of such a recovery. We conclude that the sudden change in $\dot{\nu}$ is almost certainly a state transition rather than a glitch. We will refer to the state before the transition as the "low spin-down" state and the state after the transition as the "high spin-down" state. There is also no indication of a change in the pulsed X-ray emission from B0540-69. Figure 2 shows the average pulsed count rate $\left(r_{\text {pulse }}=A \times C / t_{\exp } / N_{\mathrm{PCU}}\right.$, where $\mathrm{A}$ is the relative sine wave amplitude, $\mathrm{C}$ is the total number of counts detected, $t_{\mathrm{exp}}$ is the exposure time, and $N_{\mathrm{PCU}}$ is the average number of PCUs that were on during the observation). The average pulsed count rate for the two observations after the transition $\left(0.733 \pm 0.086 \mathrm{~s}^{-1}\right)$ is consistent with the average for the first 12 observations 


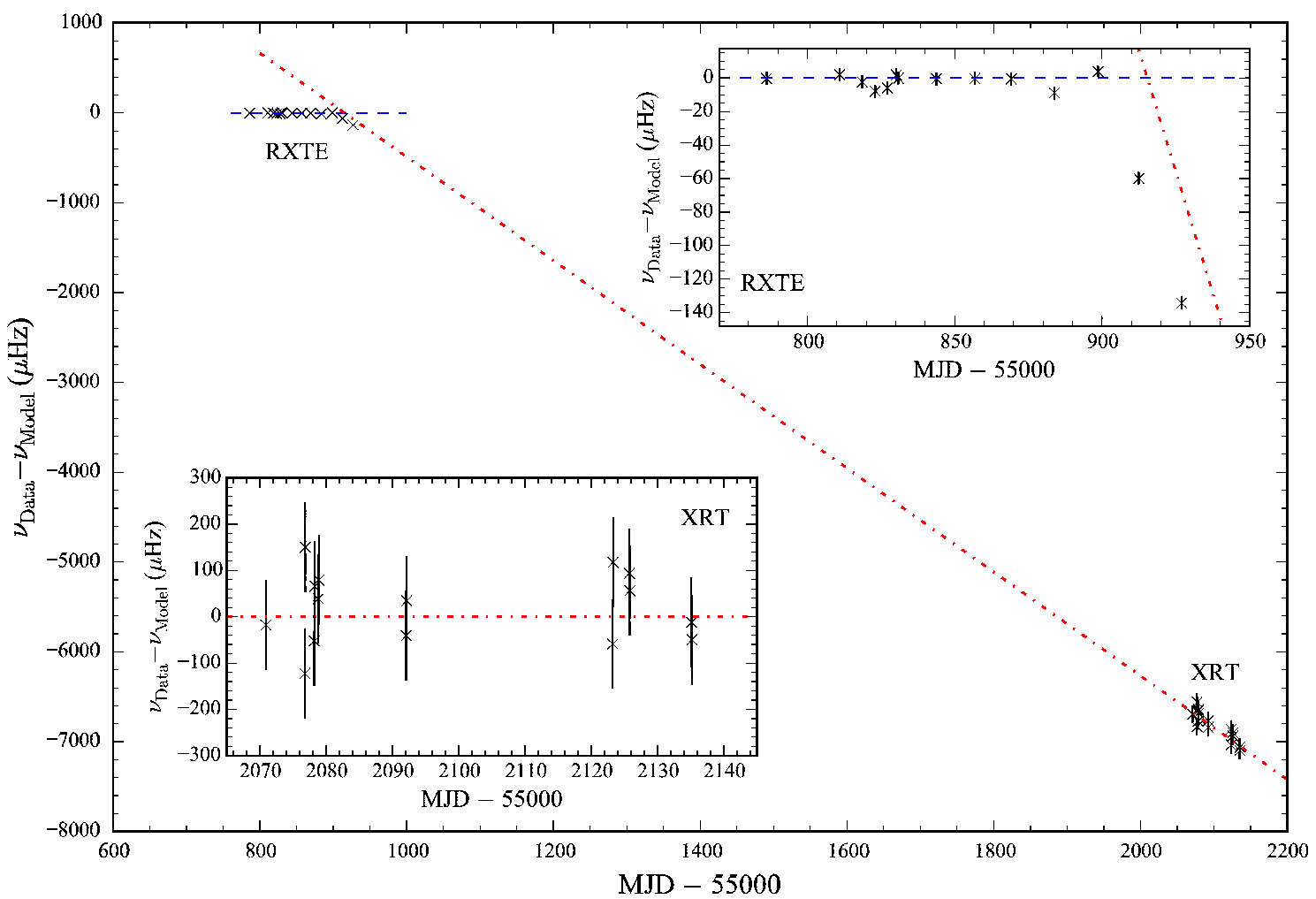

Figure 1. The frequency residuals for the RXTE and $X R T$ observations relative to the ephemeris model (the dark blue dashed line) determined with the $R X T E$ observations before the state transition. The dashed-dotted red line shows the best-fit ephemeris for the XRT data. $1 \sigma$ uncertainties are shown, but they are smaller than the symbols for the RXTE data.

Table 2

Ephemeris Parameters

\begin{tabular}{lcc}
\hline \hline Parameter & Pre-transition 2011 & 2015 \\
\hline Epoch (Modified Julian Date) & 55892.352587464 & 57077.537772223 \\
Phase & $0.000(15)$ & $0.000(23)$ \\
$\nu(\mathrm{Hz})$ & $19.72655182(1)$ & $19.70077383(4)$ \\
$\dot{\nu}\left(10^{-10} \mathrm{~s}^{-2}\right)$ & $-1.86322(2)$ & $-2.52871(8)$ \\
$\ddot{\nu}\left(10^{-21} \mathrm{~s}^{-3}\right)$ & 3.249 (fixed) & 3.249 (fixed) \\
\hline
\end{tabular}

Note. The numbers in parentheses are the $90 \%$ confidence errors quoted in the last digit.

$\left(0.718 \pm 0.035 \mathrm{~s}^{-1}\right)$. Further the folded light curves for these two observations show no significant change.

To confirm the continued nominal performance of the PCA including the final two observations, we processed the same observations for PSR J0537-6910 following the procedures given in Marshall et al. (2004). After the glitch near MJD 55815 , the final 12 observations are very well fit using the standard form for the ephemeris with an rms phase residual of $\sim 1 \%$. We conclude that the unexpected values of $\nu$ for B054069 are not due to any instrumental effect.

\subsection{Swift XRT Observations}

The XRT started observations of B0540-69 on 2015 February 17 , as part of a Target of Opportunity campaign. The $X R T$ is a focusing $X R T$ with a CCD detector with an effective bandpass of $0.3-10 \mathrm{keV}$. All observations were made using the Window Timing mode in which the central 200 CCD columns are continously read out, providing a time resolution of $1.7 \mathrm{~ms}$. The $X R T$ data were reduced with the standard software (XRTPIPELINE v0.13.1) applying the default filtering and screening criteria (HEASofT 6.16), using the 20140709 update to the XRT CALDB files and the 20150428 update to the clock correction file. The phase and frequency were measured for each continuous viewing interval (a "snapshot"). Each of the Swift entries in Table 1 except the first consists of two snapshots separated by about 96 minutes (the duration of a Swift orbit). The first entry has a single snapshot. We obtained the best ephemeris by determining a model for the closely spaced observations and then refining the model as more widely separated observations were added. All the models assumed a value of $3.249 \times 10^{-21} \mathrm{~s}^{-3}$ for $\ddot{\nu}$ based on measurements with RXTE before the state transition in 2011 . This parameter makes a very small contribution to the model because the duration of the current Swift campaign is only 65 days. The best-fit model was confirmed using a large grid search of possible combinations of $\nu$ and $\dot{\nu}$. The parameters of the best-fit model and the $90 \%$ confidence uncertainties are given in Table 2 . The model provides a good fit to the data with rms phase residuals of $\sim 3 \%$. Figure 1 , which shows the frequency residuals relative to the $R X T E$ ephemeris before the transition, displays the overall history of $\nu$.

We used two methods to determine $\dot{\nu}$ after the state transition. The first method uses the best-fit model for the $X R T$ data (Table 2) with a value of $-2.52871 \pm$ $0.00008 \times 10^{-10} \mathrm{~s}^{-2}$ at the $X R T$ epoch. After adjusting by $-3.33 \times 10^{-13} \mathrm{~s}^{-2}$ to account for the effect of $\ddot{\nu}$, this is a change in $\dot{\nu}$ from the value before the transition (Table 2) of $-0.66882 \pm 0.00008 \times 10^{-10} \mathrm{~s}^{-2}$.

The second method compares the average value of $\nu$ in the final two RXTE observations $\left(19.72601295 \mathrm{~Hz} \pm 4.6 \times 10^{-6}\right.$ $\mathrm{Hz}$ at MJD 55919.71) with the best-fit value for the $X R T$ data 


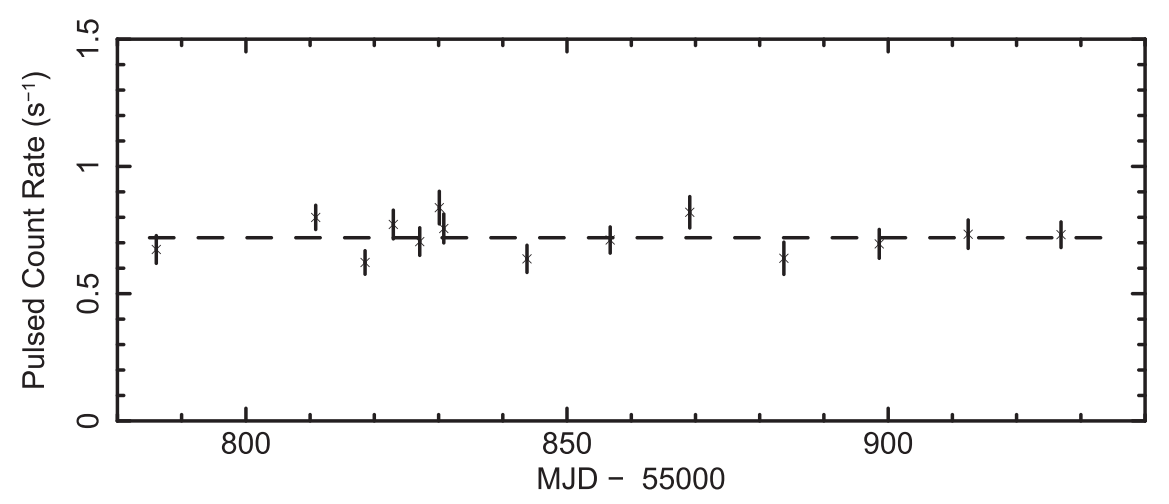

Figure 2. Pulsed count rate with $1 \sigma$ uncertainties for the RXTE observations.

of $19.70077383 \mathrm{~Hz} \pm 4 \times 10^{-8} \mathrm{~Hz}$. The resulting $\dot{\nu}$ is $-2.5230 \pm 0.0005 \times 10^{-10} \mathrm{~s}^{-2}$ at MJD 56498.62. After adjusting by $1.62 \times 10^{-13} \mathrm{~s}^{-2}$ to account for the effect of $\ddot{\nu}$, the result is $-2.5214 \pm 0.0005 \times 10^{-10} \mathrm{~s}^{-2}$ at the XRT epoch, which is larger than result from the first method by $7.3 \pm 0.5 \times 10^{-13} \mathrm{~s}^{-2}$. This discrepancy is shown in Figure 1 in which the extrapolation of the $X R T$ ephemeris back to the final two RXTE observations predicts a value for $\nu$ that is $7.3 \times 10^{-5} \mathrm{~s}^{-1}$ larger than that observed. The uncertainty of the extrapolated values due to the uncertainty in the model's $\dot{\nu}$ is only $8 \times 10^{-7} \mathrm{~Hz}$. The discrepancy suggests that the evolution of the high spin-down state is more complicated than our simple model. The second method indicates a change in $\dot{\nu}$ from the value before the transition of $-0.6615 \pm$ $0.0005 \times 10^{-10} \mathrm{~s}^{-2}$.

Since the value of $\ddot{\nu}$ after the state transition is uncertain and the statistical uncertainties are small, we use the value of the adjustments due to $\ddot{\nu}$ to estimate the uncertainty. The relative change in $\dot{\nu}$ is then $35.9 \% \pm 0.2 \%$ and $35.5 \% \pm 0.1 \%$ for the first and second methods respectively. We adopt $35.7 \% \pm$ $0.4 \%$, the average of the two methods with an uncertainty that encompasses the individual error bars, as our best estimate of the relative change in $\dot{\nu}$.

The folded light curve is similar to that reported from other X-ray observations (e.g., Cusumano et al. 2003). Although the actual light curve is more complicated, a model of a constant plus a Gaussian provides a good description of the XRT data. The best-fit standard deviation for the Gaussian is $0.174 \pm$ 0.022 , which is consistent with a fit to the folded light curve for the RXTE data before the state transition.

\section{DISCUSSION}

B0540-69 has been extensively monitored by numerous observatories in the more than 30 years since its discovery including Ginga observations spanning 4.4 years (Deeter et al. 1999) and the 12.5 years of observations with RXTE. More than 10 measurements of the braking index for the pulsar, which require long spans of data, have been reported (Cusumano et al. 2003, who list previous measurements; Ferdman et al. 2015). Values for the braking index range from 1.81 (Zhang et al. 2001) to 2.74 (Ögelman \& Hasinger 1990).

None of these studies found a sudden change in $\dot{\nu}$ comparable to the one reported here. The glitches in $\dot{\nu}$ reported by Zhang et al. (2001) and Ferdman et al. (2015) are 2000 times smaller, and knowledge of the pulsar's phase is maintained throughout the 12.5 years of RXTE monitoring until the final two observations. When discovered with the Einstein Observatory (Seward et al. 1984), B0540-69 had a $\dot{\nu}$ of $-1.900 \times 10^{-10} \mathrm{~Hz} \mathrm{~s}^{-1}$, which indicates that the pulsar was in the low spin-down state in 1979 and 1980. The lack of published values of $\dot{\nu}$ indicative of the high spin-down state suggests that B0540-69 remained in the low spin-down state the vast majority of the time, if not all the time, until the transition in late 2011.

The sudden change in the spin-down rate is most likely associated with a change in the magnetosphere of the NS. One possibility is a global change in the conductivity of the magnetosphere. Li et al. (2012) developed solutions for pulsar magnetospheres with finite resistivity that mitigate some of the limitations of the vacuum and force-free models. The dependence of the spin-down luminosity was calculated for a wide range of the conductivity parameter $\sigma$ (expressed in terms of the pulsar angular rotation rate $\Omega$ ) and inclination angle $\alpha$ using a three-dimensional numerical code. For broad ranges of $\alpha\left(0^{\circ}-90^{\circ}\right)$ and the conductivity $\left((\sigma / \Omega)^{2}\right.$ from 0.04 to 4.0$)$, a $36 \%$ increase in the luminosity (and by extension in $|\dot{\nu}|$ ) can be achieved with an increase in $\sigma$ of less than a factor of 6 . There are maximum values of $(\sigma / \Omega)^{2}$, ranging from 40 at $\alpha=0^{\circ}$ to 1.3 at $\alpha=90^{\circ}$ above which it is no longer possible to explain an increase in the luminosity with an increase in $\sigma$. Since for large values of $(\sigma / \Omega)$, the component of the electric field parallel to the magnetic field $E_{\|} \propto \sigma^{-1}$ (Kalapotharakos et al. 2014), an increase in $\sigma$ may reduce the gamma-ray flux from the pulsar. The long-term light curve of B0540-69 in the energy range of $200 \mathrm{MeV}-100 \mathrm{GeV}$ (Fermi-LAT Collaboration 2015, in preparation) shows no indication of change in the gammaray flux with an upper limit of $30 \%$. Detailed modeling, such as that done by Brambilla et al. (2015) for other Fermi pulsars, is needed to understand the implication of this constraint on parameters for B0540-69 but this is beyond the scope of this paper.

Another possible explanation for the spin-down transition is a change in torque due to a change in the plasma outflow from the polar caps. Kramer et al. (2006) suggested this to explain the state transitions in the intermittent pulsar PSR B1931+24. Unlike PSR B1931+24, B0540-69 appears to be radio bright in its low spin-down state, but its radio flux may significantly increase or its pulse shape may change in the high spin-down state. Following Young et al., we estimate the change in the charge density $\rho_{\text {plasma }}$ as $7.1 \times 10^{5} \Delta \dot{\nu} \nu^{-0.5} \dot{\nu}_{\text {low }}^{-0.5} \mathrm{C} \mathrm{m}^{-3}$, assuming a pulsar radius of $10 \mathrm{~km}$ and $I_{45}$ of 1 . This estimate of $0.78 \mathrm{C} \mathrm{m}^{-3}$ is close to the Goldreich-Julian charge density for B0540-69 of $1.09 \mathrm{C} \mathrm{m}^{-3}$. An increase in $\rho_{\text {plasma }}$ predicts an 
increase in the radio flux, which can be tested with radio observations of the high spin-down state. It also predicts a reduction in the braking index as the relative importance of magnetic dipole radiation decreases.

A change in the amount of open poloidal magnetic flux would also change the spin-down luminosity, which is expected to be proportional to $r_{\text {open }}^{-2}$ where $r_{\text {open }}$ is the radius beyond which the magnetic field lines become open (Contopoulos 2007). A $36 \%$ increase in the spin-down luminosity with no change in $\nu$ would require a $15 \%$ decrease in $r_{\text {open }}$.

\section{SUMMARY}

The spin-down rate of B0540-69 increased suddenly by $36 \%$ (a change in $\dot{\nu}$ of $-6.7 \times 10^{-11} \mathrm{~Hz} \mathrm{~s}^{-1}$ ) between 2011 December 3 and 17. Observations with Swift in 2015 show that this change in spin-down rate has persisted for more than 3 years. Such a large persistent change in the spin-down rate has never been reported for B0540-69. There is no indication of a simultaneous change in the pulse shape in X-rays, the pulsed X-ray luminosity, or the total gamma-ray luminosity. We interpret this change as a transition between two stable states of the pulsar, similar to state transitions in other pulsars. B0540-69 extends the class of state changing pulsars to include a very young and luminous example. The transitions in B0540-69 appear to be rare with the low spindown state probably lasting more than 30 years. Future work can test predicted changes in the radio flux and the braking index in the high spin-down state.

This work made use of data supplied by the UK Swift Science Data Centre at the University of Leicester and the High Energy Astrophysics Science Archive Research Center, provided by NASA's Goddard Space Flight Center.

\section{REFERENCES}

Allafort, A., Baldini, L., Ballet, J., et al. 2013, ApJL, 777, L2

Anderson, P. W., \& Itoh, N. 1975, Natur, 256, 25

Bradt, H. V., Rothschild, R. E., \& Swank, J. H. 1993, A\&AS, 97, 355

Brambilla, G., Kalapotharakos, C., Harding, A., \& Kazanas, D. 2015, ApJ, 804, 84

Burrows, D. N., Hill, J. E., Nousek, J. A., et al. 2005, SSRv, 120, 165

Camilo, F., Ransom, S. M., Chatterjee, S., Johnston, S., \& Demorest, P. 2012, ApJ, 746, 63

Contopoulos, I. 2007, A\&A, 466, 301

Cusumano, G., Massaro, E., \& Mineo, T. 2003, A\&A, 402, 647

Deeter, J. E., Nagase, F., \& Boynton, P. E. 1999, ApJ, 512, 300

Espinoza, C. M., Lyne, A., Stappers, B. W., \& Kramer, M. 2011, MNRAS, 414, 1679

Ferdman, R. D., Archibald, R. F., \& Kaspi, V. M. 2015, ApJ, submitted (arXiv:1506.00182)

Franco, L., Link, B., \& Epstein, R. I. 2000, ApJ, 543, 987

Gehrels, N., Chincarini, G., Giommi, P., et al. 2004, ApJ, 611, 1005

Kalapotharakos, C., Harding, A., \& Kazanas, D. 2014, ApJ, 793, 97

Kramer, M., Lyne, A. G., O’Brien, J. T., Jordan, C. A., \& Lorimer, D. R. 2006, Sci, 312, 549

Li, J., Spitkovsky, A., \& Tchekhovskoy, A. 2012, ApJ, 746, 60

Livingstone, M. A., Kaspi, V. M., \& Gavriil, F. P. 2005, ApJ, 633, 1095

Lorimer, D. R., Lyne, A. G., McLaughlin, M. A., et al. 2012, ApJ, 758, 141

Lyne, A. G., Hobbs, G., Kramer, M., Stairs, I., \& Stappers, B. W. 2010, Sci, 329,408

Lyne, A. G., Jordan, C. A., Graham-Smith, F., et al. 2015, MNRAS, 446, 857

Manchester, R. N., Mar, D. P., Lyne, A. G., Kaspi, V. M., \& Johnston, S. 1993, ApJL, 403, L29

Marshall, F. E., Gotthelf, E. V., Middleditch, J., Wang, D. Q., \& Zhang, W. 2004, ApJ, 603, 682

Mignani, R. P., Sartori, A., De Luca, A., et al. 2010, A\&A, 515, 110

Ögelman, H., \& Hasinger, G. 1990, ApJL, 353, L21

Rots, A. H., Jahoda, K., Macomb, D. J., et al. 1998, ApJ, 501, 749

Seward, F. D., Harnden, F. R., \& Helfand, D. J. 1984, ApJL, 287, L19

Young, N. J., Stappers, B. W., Lyne, A. G., et al. 2013, MNRAS, 429, 2569

Yu, M., Manchester, R. N., Hobbs, G., et al. 2013, MNRAS, 429, 688

Zhang, W., Marshall., F. E., Gotthelf, E. V., Middleditch, J., \& Wang, Q. D. 2001, ApJL, 554, L177 